Article

\title{
Microfluidic Induced Controllable Microdroplets Assembly in Confined Channels
}

\author{
Juan Wang, Mingliang Jin, Tao He, Guofu Zhou and Lingling Shui * \\ Institute of Electronic Paper Displays, South China Academy of Optoelectronics, \\ South China Normal University, Guangzhou 510006, China; E-Mails: wj8686054@gmail.com (J.W.); \\ jinml@scnu.edu.cn (M.J.); taohewhh@gmail.com (T.H.); zhougf@ scnu.edu.cn (G.Z.) \\ * Author to whom correspondence should be addressed; E-Mail: Shuill@m.scnu.edu.cn; \\ Tel./Fax: +86-20-3931-4813.
}

Academic Editors: Andrew deMello and Xavier Casadevall i Solvas

Received: 25 July 2015 / Accepted: 1 September 2015 / Published: 10 September 2015

\begin{abstract}
We report on the microfluidic induced monodispersed microdroplet generation and assembly in confined microchannels. Two and three dimensional close-packed droplet lattices were obtained in microfluidic devices by adjusting the channel geometry, the fluidic flow rates and the monodispersed droplet size. The droplet packing was mainly caused by the volumetric effect and capillarity in confined microchannels. Polymerizable fluids were also investigated to demonstrate the effect of fluidic properties on the microdroplet generation and assembly, which could find interesting applications in the future. This approach would be helpful to fundamentally understand the mechanism of self-assembly process of particles in confined microstructures, and practically be applied in sensing and energy storage devices.
\end{abstract}

Keywords: microfluidics; microdroplet; self-assembly; close-packed configuration

\section{Introduction}

Self-assembly happens in nature with various forms induced by physical, chemical or biological forces [1-3]. Self-assembly refers to a rearrangement process of the entities like molecules, macromolecules, colloidal particles (gas-bubbles, liquid-droplets or solid-particles) because of the free energy minimization of a system, leading to a transition from a non-equilibrium state to an equilibrium state. Direct self-assembly implies an approach to precisely control over rearrangement process to obtain their devised and desired self-organization structures and configurations, while retaining the key 
elements of spontaneous self-organization [4,5]. Materials with self-assembled structures have received tremendous and increasing attention because of their potential applications in various fields, such as sensors [6], electrical or optical switchers [7], photonic devices [8], data storage materials [9] and bioassays [10].

Fluid-based components represent a class of components that are intrinsically well suited for dynamic and reversible control over viscosity, surface tension and optical properties. Combination of fluidics and microstructures has enabled wide applications in optical, chemical and biomedical research [11-15]. Microfluidics has been demonstrated to be a powerful platform with the capability of creating numerous monodispersed soft particles (bubbles or droplets) with controllable size, shell thickness, shapes, compartments and chemical compositions [16-19]. Moreover, in the microfluidic device, the fluidic properties and flow profiles, the microstructure size and shapes could be tuned properly. Therefore, the self-assembly process could be induced by capillary force or hydrodynamic force inside the microfluidic devices [10,20,21].

The self-assembled close-packed configurations of micro- and/or nano-structures possess the properties of high surface area and enhanced optoelectrical resonance, which are potentially required in gratings, micro-lens, photonic devices, displays, bioassays and sensors [22-27]. Choil et al. presented the self-assembly procedure of the silica microparticles in microfluidic channels due to the capillarity of the microparticles dispersed suspension [28]. Binary opal lattices have been obtained owing to the capillary force and surface tension in the microfluidic device by Malekpourkoupaei and colleagues [20]. Park and co-workers have demonstrated a microfluidic approach to self-assemble colloidal particles at the gas-liquid interface by varying the $\mathrm{pH}$ value of the interface [29]. Monodispersed photonic electro-responsive Janus microspheres have been created with the assistance of a microfluidic device, showing structural colors of the nanoparticle crystals [24]. Furthermore, highly ordered structures of fluidic particles (gas-bubbles or liquid-droplets) have been observed in microfluidic devices, which could afford unique fluidic hydrodynamics and boundary restriction, and be applied for imaging, bioassaying, data storaging and environment monitoring [10,21,22,30,31].

Microfluidic devices allow sensitive control over fluidic components and flow rates. Therefore, the process and parameters of fluidic particles generation and assembly could be precisely controlled. The close-packed bubble/droplet lattices have been created by controlling the volume flow ratio of the entrance air or liquid [14,21]. In these devices, the bubbles/droplets generation and assembly took place at the same time by controlling the volume flow ratio. The soft particle size and packing configurations were determined by the same entrance flows, which was therefore difficult to control independently [21]. In this article, we designed a microfluidic device for microdroplets generation and assembly in different areas of the device. Therefore, the droplet generation and assembly could be tuned separately with better controlling over droplet size and different assembly configurations in one device. The capillary force together with the volumetric effect and hydrodynamic force determined the droplet generation and assembly in the confined channels. The flow rates and ratios as well as the fluidic properties were investigated according to their effects on assembly configurations. Additionally, the photocurable liquids of $\mathrm{N}$-isopropylacrylamide (NIPAM) aqueous solutions were also employed in this system. We expect that the close-packed fluidic structures could be on-line solidified, obtaining solid-based well-ordered close-packed configurations which would be more promising for applications as optical components, 
catalysis templates, micro-lens and light collection devices. Taking the advantages of microfluidics, the physical and chemical properties of the dispersions could be precisely controlled, which would also help to deeply understand the self-assembly process.

\section{Experimental Section}

\subsection{Materials}

$N$-hexadecane (99\%) and sorbitan monooleate (Span 80) were purchased from Acros Organics (Geel, Belgium) and Aladdin (Shanghai, China), respectively. Hexadecane mixed with surfactant of Span 80 was acted as the continuous phase (oil phase) in the microfluidic device. Mineral oil and ABIL EM 90 (Bis-PEG/PPG-14/14 Dimethicone, Cyclopentasiloxane, a silicon based water-in-oil emulsifier) were purchased from J \& K (Beijing, China) and Evonik Degussa (Essen, Germany), respectively. Mineral oil contained the surfactant of ABIL EM 90 was served as the continuous phase (oil phase) in poly(dimethylsiloxane) (PDMS) microfluidic device. $N$-isopropylacrylamide (NIPAM, 99\%) was purchased from Acros Organics, which was used as the photocurable monomer. $N, N$-methylenebisacrylamide (MBA, 99\%) was purchased from Sigma-Aldrich (St. Louis, MO, USA) and 2-hydroxy-4-(2-hydroxyethoxy)-2-methylpropiophenone (I 2959, >98\%) was purchased from Tokyo Chemical Industry (TCI) (Shanghai, China), which were used as the cross-linker and water-soluble photoinitiator, respectively. Deionized (DI) water $\left(18.2 \mathrm{M} \Omega\right.$ at $\left.25^{\circ} \mathrm{C}\right)$ was prepared from a water purification system (Water Purifier, Sichuan, China). PDMS (Sylgard 184) package consisted of a base and curing agent was purchased from Dow Corning Corporation (Midland, MI, USA). The SU-8 3050 photoresist and developer solution were purchased from MicroChem (Westborough, MA, USA).

\subsection{Design and Fabrication of Poly(dimethylsiloxane) (PDMS) Microfluidic Devices}

The geometry of the microfluidic device is shown in Figure 1. The device includes two sections (I and II): Section I is for creating water-in-oil (W/O) microdroplets and section II is to induce assembly of the generated microdroplets in the main-channel. "a" and "b" are the inlets of the continuous phase (oil phase) and dispersed phase (water phase), respectively. The flow-focusing position is located at " 3 " and connected to the self-assembly section. " 4 " is the main outlet and the confinement channel for droplets assembly. The side-channel " 6 " is for taking away the continuous oil phase from "4" to "5". "e" and "d" are the outlets for "4" and "5", respectively. Figure 1B is an optical microscopic image of the experimentally fabricated device. In section I, the width of the water entrance and oil entrance are both $400 \mu \mathrm{m}$. The width of the flow-focusing location $\left(w_{\mathrm{f}}\right)$ is either 25 or $50 \mu \mathrm{m}$ in our experiment. In section II, the side-channel width $\left(w_{\mathrm{o}}\right)$ is $10 \mu \mathrm{m}$ which is smaller than the generated microdroplets diameter. The length of the side-channel and main assembly channel are $500 \mu \mathrm{m}$ and $1.4 \mathrm{~cm}$, respectively. The number of the side-channel designed is 250 , which is enough to take away the oil from the assembly channel " 4 " to the outside channel " 5 ", resulting in the monodispersed microdroplets packing into well-ordered arrangements. The depth of the microfluidic channels is about $40 \mu \mathrm{m}$.

The PDMS-based microfluidic device was fabricated by using soft lithography [32]. The CAD (Computer-Aided Design) drawing was transferred and patterned onto a chrome plate as a photomask. 
The mold (master) was fabricated on a silicon wafer using SU-8 3050 photoresist. A mixture of the PDMS pre-polymer and curing agent at the mass ratio of 10:1 was thoroughly stirred and degassed. The degassed mixture was then poured onto the mold and cured at $80^{\circ} \mathrm{C}$ for $1 \mathrm{~h}$ in an oven. The PDMS replica with designed structures was then peeled from the silicon master. Connection holes were drilled through the PDMS before bonding. In the end, the PDMS replica with microfluidic channels and a piece of clean glass slide were oxygen plasma treated for $50 \mathrm{~s}$ and bonded to each other. The detailed device fabrication process was shown in Figure S1. The experimental setup for microdroplets fabrication and assembly was presented in Figure S2.
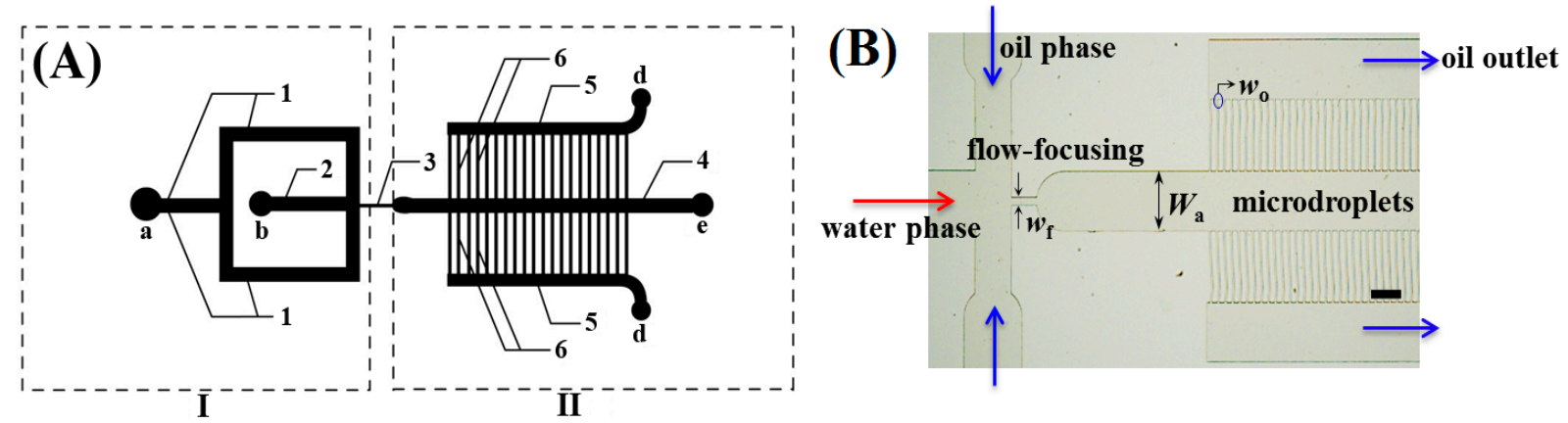

Figure 1. (A) Schematic drawing of the flow-focusing microfluidic device with section I and section II for droplets generation and assembly; (B) Optical microscopic image of the fabricated poly(dimethylsiloxane) (PDMS) device. " $w_{\mathrm{f}}$ ", " $W_{\mathrm{a}}$ " and " $w_{\mathrm{o}}$ " are the width of the flow-focusing channel, main assembly channel and side-channel, respectively. The scale bar is $200 \mu \mathrm{m}$.

\subsection{Microfluidic Manipulation and Characterization}

The oil phase and water phase were introduced into the microfluidic device correspondingly via the inlets through microtubes by two syringe pumps (LSPO2-1B, Longer, Baoding, China), as shown in Figure 1B. The water phase was sheared off and formed monodispersed microdroplets by the oil phase at the flow-focusing area " 3 ". The water phase used in the experiment was deionized (DI) water or DI water consisted of NIPAM, MBA and I 2959; the oil phase was hexadecane with Span 80 or mineral oil contained ABIL EM 90.

The water-in-oil (W/O) droplets generation and their on-line assembly driven by the fluidic flow through side-channels were visualized and recorded using an inverted optical microscope (Olympus IX2, Tokyo, Japan) equipped with a high-speed camera (Phantom Miro M110, Vision Research Inc., Wayne County, NC, USA). Bright-field images were recorded by the high-speed camera mounted on the inverted optical microscope. The interfacial tension of oil-water was measured using DCAT21 tensionmeter (DataPhysics Instruments GmbH, Filderstadt, Germany). The size of the microdroplets was analyzed by Image-Pro Plus 6.0 software.

\section{Results and Discussion}

In this work, different oil-water two-phase systems were exploited for droplets generation and assembly in the microfluidic device. The two-phase system of hexadecane with $2.7 \%(w / v)$ Span 80 
and DI water was chosen as example to investigate and obtain experimental parameters. Moreover, the photocurable NIPAM aqueous solution was investigated to explore the effect of fluidic properties on the droplets generation and assembly in the confined microchannels, which might be helpful to understand the materials selection criteria for practical applications.

\subsection{Preparation of Monodispersed Water-in-Oil (W/O) Microdroplets}

The monodispersed W/O emulsion microdroplets were generated in the PDMS-based flow-focusing microfluidic devices owing to the wettability of the microchannel walls [33]. The oil phase and water phase were pumped into their corresponding microchannels using syringe pumps. The highly monodispersed microdroplets were obtained at the flow-focusing location, as illustrated in Figure 2A. Hexadecane with $2.7 \%(w / v)$ Span 80 and DI water were used as the oil phase and water phase, respectively. By controlling the two phases volume flow rates and ratios, microdroplets with different diameters in the range of $20-50 \mu \mathrm{m}$ have been obtained in the devices. The generated microdroplets had a narrow size distribution with the coefficient of variation $(\mathrm{CV}, \%)<2.0$, as shown in Figure $2 \mathrm{~B}$.

The microdroplets fabrication was subjected to several effects such as fluidic flow rates, viscosity, wettability, surface intension and the microchannel geometry [34]. Generally, in a microfluidic device, the determinative driving forces are interfacial tension force, viscous force and the fluidic channel properties [35,36]. The dimensionless number of $\mathrm{Ca}$ (Capillary number) has been used to describe two-phase flow in microchannels, which is defined as:

$$
C a=\frac{\eta v}{\sigma}
$$

where $\eta$ is the viscosity of the continuous phase $(\mathrm{Pa} \cdot \mathrm{s}), v$ is the average flow velocity $\left(\mathrm{m} \cdot \mathrm{s}^{-1}\right)$, and $\sigma$ is the interfacial tension $\left(\mathrm{N} \cdot \mathrm{m}^{-1}\right)$ between oil and water. $C a$ is typically used to explain the microfluidics phenomena, especially for the generation of microdroplets. When $C a \ll 1$, the interfacial tension is dominant, leading to the formation of droplets [37].

In our experiment, the fluidic properties were kept constant, the effect of the volume flow rate of each phase on the microdroplets diameter in dripping region [38-40] was explored as shown in Figure 2C,D. The microfluidic device with $w_{\mathrm{f}}=25 \mu \mathrm{m}$ was used to fabricate monodispersed emulsion droplets. $Q_{\mathrm{o}}$ and $Q_{\mathrm{w}}$ were expressed as the volume flow rate of the oil phase and water phase, respectively. When $Q_{\mathrm{w}}$ was kept constant, the average diameter of the microdroplets decreased as $Q_{\mathrm{o}}$ increased within a range of flow rates, where the value of $\mathrm{Ca}$ increased as well, as demonstrated in Figure 2C. On the contrary, the average diameter of the spherical microdroplets increased with $Q_{\mathrm{w}}$ and $C a$, when $Q_{\mathrm{o}}$ was constant, as shown in Figure 2D. The relationship of microdroplets average diameter to $\mathrm{Ca}, Q_{\mathrm{o}}$ and $Q_{\mathrm{w}}$ was also explored in the microfluidic device with $w_{\mathrm{f}}=50 \mu \mathrm{m}$ in different microdroplets generation regions. The similar curve trend was observed for two devices, revealing each phase flow effect on the microdroplet size.

Over a wide range of flow rates, $Q_{\mathrm{o}}$ in the range of $40-800 \mu \mathrm{L} \cdot \mathrm{h}^{-1}$ and $Q_{\mathrm{w}}$ in the range of 10-200 $\mu \mathrm{L} \cdot \mathrm{h}^{-1}$, the two-phase (oil-water) droplet-based flow diagram revealed different flow regions in response to the volume flow rates, as shown in Figure 3. The dripping mode of microdroplet formation was observed at low flow rates. By increasing the flow rates, the microdroplet formation experienced a transition from a dripping to jetting region. Monodispersed microdroplet generation in the dripping (i) and jetting region (iii) were demonstrated in Figure 3B. 

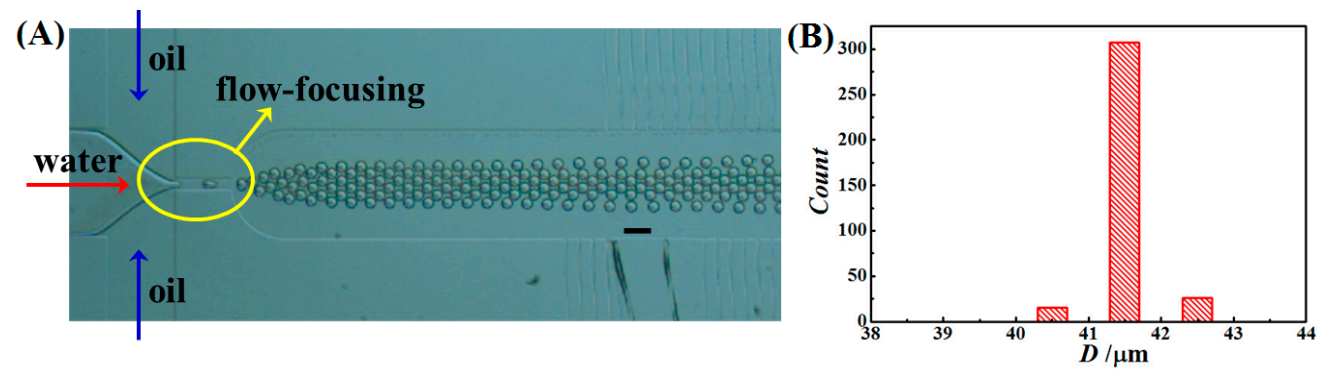

(C)

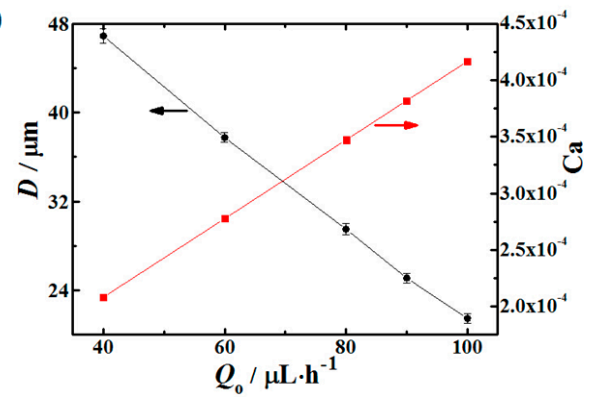

(D)

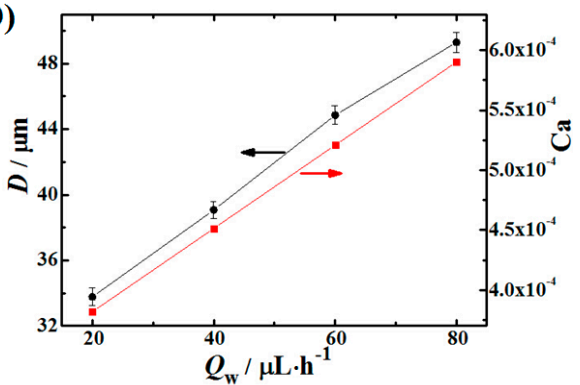

Figure 2. (A) An optical microscopic image of the microdroplets generation in a PDMS-based flow-focusing microfluidic device. The scale bar is $100 \mu \mathrm{m}$. (B) The size distribution of the microdroplets generated in the device $(\mathbf{A})$. The average diameter of the microdroplets was $41.5 \mu \mathrm{m}$ with the coefficient of variation $(\mathrm{CV}) 0.7 \%$. In (A) and (B), the highly uniform microdroplets were generated in the device with $w_{\mathrm{f}}=50 \mu \mathrm{m}$. (C) The microdroplets diameter changed with $C a$ and $Q_{\mathrm{o}}$ in dripping region when $Q_{\mathrm{w}}$ was fixed at $20 \mu \mathrm{L} \cdot \mathrm{h}^{-1}$. $Q_{\mathrm{o}}$ was varied from 40 to $100 \mu \mathrm{L} \cdot \mathrm{h}^{-1}$. (D) The microdroplets diameter changed with $C a$ and $Q_{\mathrm{w}}$ in dripping region when $Q_{\mathrm{o}}$ was kept constant at $90 \mu \mathrm{L} \cdot \mathrm{h}^{-1} \cdot Q_{\mathrm{w}}$ was varied in the range of $20-80 \mu \mathrm{L} \cdot \mathrm{h}^{-1}$. In (C) and (D), these monodispersed microdroplets were obtained in the device with $w_{\mathrm{f}}=25 \mu \mathrm{m}$. The oil phase and water phase were hexadecane with $2.7 \%(w / v)$ Span 80 and DI water, respectively.
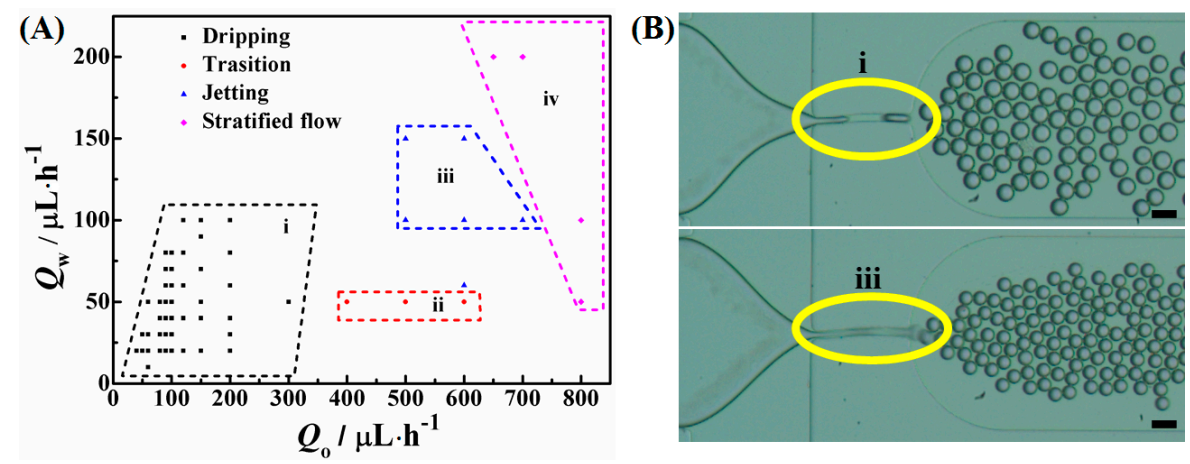

Figure 3. (A) Diagram of the microdroplets formation modes as a function of volume flow rates of oil and water phase. The modes were divided into (i) dripping-flow, (ii) transition-flow (from dripping to jetting), (iii) jetting-flow and (iv) stratified-flow; (B) Optical microscopic images of microdroplets generation under dripping (i) and jetting (iii) mode. Scale bars are $50 \mu \mathrm{m}$. $Q_{\mathrm{o}}$ and $Q_{\mathrm{w}}$ were 80 and $20 \mu \mathrm{L} \cdot \mathrm{h}^{-1}$ for top image of (B), 500 and $150 \mu \mathrm{L} \cdot \mathrm{h}^{-1}$ for bottom image of (B). Hexadecane with $2.7 \%(w / v)$ Span 80 and DI water were used as the oil and water phase, respectively, to generate the emulsion droplets through the same microfluidic device with $w_{\mathrm{f}}=25 \mu \mathrm{m}$. 


\subsection{Microdroplet Assembly in the Restricted Microchannels}

Monodispersed microdroplets flowed into section II of the microfluidic device after generating, and assembled into close-packed arrangements in the confined main-channel as the continuous phase was squeezed out from the side-channels. The continuous phase flowed through side-channels according to the capillary force and the pressure difference between two ends of the side-channels. In the main-channel, the volume of the channel was constant, and the volume ratio of droplets to the confined channel gradually increased with continuous phase flowing away. The microdroplets then rearranged themselves in an ordered way to minimize the free energy of the system.

The monodispersed microdroplets self-assembled into 2D and 3D configurations in the confined channel of the microfluidic devices. Well-ordered close-packed single-layer hexagonal arrangements and double-layer square or hexagonal configurations have been observed in a wide range of flow rates at different microfluidic devices, as shown in Figure 4. It is well known that the spherical particles self-assemble into close-packed arrangements when the volume fraction of particles in a confined volume increases in order to minimize the free energy. In a microfluidic device, both continuous and dispersed phase flow can be controlled; therefore, the volume fraction can be controlled by varying flow rates and channel geometry. On the other hand, the droplet size and shearing force induced by fluidic flow plays a significant role on the droplets packing. Several parameters normally co-determined the microdroplet assembly configurations. In this report, we investigated the effects of the location of the droplets in the main-channel, two phase volume flow rates, droplet size and the water phase properties on the droplets assembly configurations in the microfluidic channel.

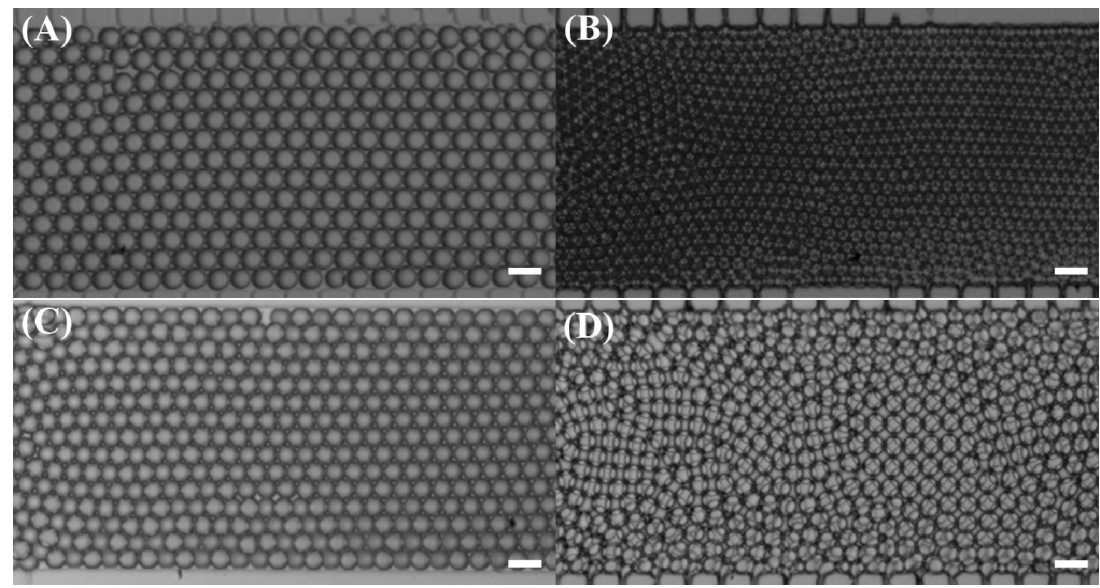

Figure 4. Optical microscopic images of the microdroplets assembly configurations at various flow rates and at different microfluidic devices with $(\mathbf{A})$ and $(\mathbf{C})$ in close-packed single-layer mode, $(\mathbf{B})$ and $(\mathbf{D})$ in close-packed double-layer mode. In (A) and (B), the microdroplets were generated in the same microfluidic device with $w_{\mathrm{f}}=25 \mu \mathrm{m}$; in $(\mathbf{C})$ and (D), the microdroplets were formed in the same microfluidic device with $w_{\mathrm{f}}=50 \mu \mathrm{m}$. In (A) and (C), the microdroplets were generated in dripping region; in (B) and (D), the microdroplets were formed under jetting mode. $Q_{\mathrm{o}}$ and $Q_{\mathrm{w}}$ were 90 and $20 \mu \mathrm{L} \cdot \mathrm{h}^{-1}$ (A), 600 and $60 \mu \mathrm{L} \cdot \mathrm{h}^{-1}$ (B), 800 and $100 \mu \mathrm{L} \cdot \mathrm{h}^{-1}(\mathbf{C}), 2000$ and $400 \mu \mathrm{L} \cdot \mathrm{h}^{-1}$ (D). The oil phase and water phase were all hexadecane with $2.7 \%(w / v)$ Span80 and DI water. Scale bars are $50 \mu \mathrm{m}$. 


\subsubsection{Microdroplet Assembly along the Confinement Main-Channel}

When the flow rate and ratio were controlled in a specific range, we found that different droplet configurations (single-layer and double-layer) were obtained at the same time along the same main-channel but at different locations. As shown in Figure 5, the produced microdroplets experienced from random arrangements to close-packed single-layer and then double-layer configurations gradually along the main assembly channel. As the oil phase was squeezed into the side-channels, the volume fraction of water droplets to oil phase increased. Here, the volume fraction $\left(\Phi_{\mathrm{vol}}\right)$ was defined as the volume ratio of the droplets to the assembly confinement microchannel, which was equal to the total volume of oil and water phase. The microdroplets flowed directly into section II after generating, and started to accumulate into random configurations (close pack without ordering) when $\Phi_{\text {vol }}$ was more than 0.19. As $\Phi_{\mathrm{vol}}$ increased to 0.27 , the droplets started packing into well-ordered structures in one layer. When $\Phi_{\mathrm{vol}}$ approached 0.53 , the droplets further packed into two-layer configurations. As the volume fraction further increased, the droplets fully filling the main-channel with oil phase only existed among the curved interface between droplets. If the value of $\Phi_{\mathrm{vol}}$ increased further, the droplets would be even well-packed but deformed at the end. The on-line packing of these microdroplets was mainly attributed to the volumetric effect. The flowing of oil phase through side-channels was according to the capillarity and the pressure difference. Because the surface of the microchannel was wetting to the oil phase, as soon as the oil phase came into contact with the side-channels area, the oil phase was imbibed into them owing to the capillary force. The exact flow of the oil phase through the side-channels corresponded to the side-channel number, size and pressure difference between the entrance and exit. Movie S1 in the supplementary information showed a complete process of the microdroplets generation and assembly along the main-channel. In the next sections, the named assembly configurations were all referred to the final equilibrium states in the end of the main-channel.
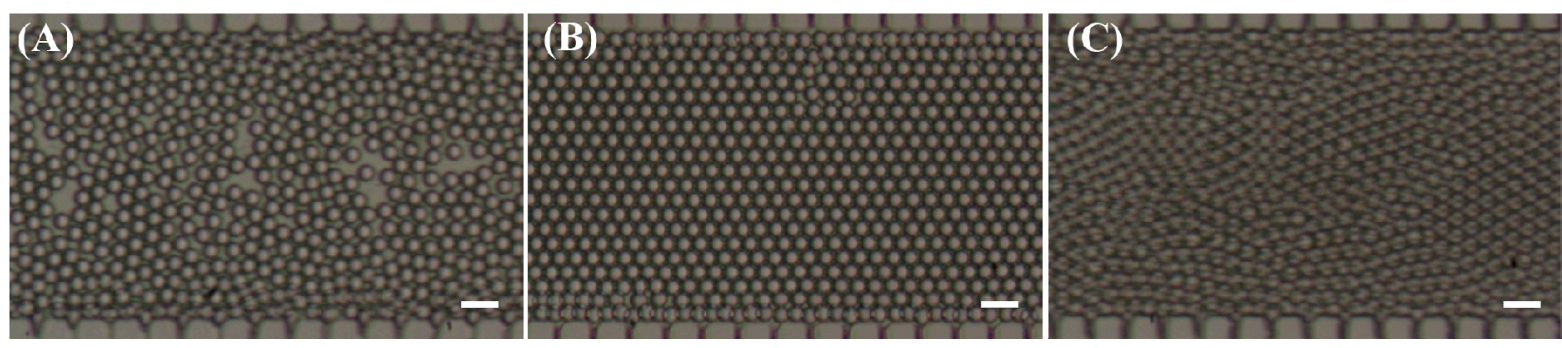

Figure 5. Series of the optical microscopic images of the monodispersed microdroplets assembly configurations at different locations along the same main-channel: (A) random arrangement, (B) close-packed single-layer configuration and $(\mathbf{C})$ close-packed double-layer configuration. The characteristic size of the microfluidic device: $w_{\mathrm{f}}=25 \mu \mathrm{m}, d_{\mathrm{c}}=40 \mu \mathrm{m}$ and $W_{\mathrm{a}}=400 \mu \mathrm{m}$. Hexadecane with $2.7 \%(w / v)$ Span 80 and DI water were used as the oil phase and water phase, respectively. Microdroplets were produced under jetting mode, with $Q_{\mathrm{o}}=600 \mu \mathrm{L} \cdot \mathrm{h}^{-1}$ and $Q_{\mathrm{w}}=100 \mu \mathrm{L} \cdot \mathrm{h}^{-1}$. Scale bars are $50 \mu \mathrm{m}$.

\subsubsection{Effect of Volume Flow Rates on the Microdroplets Assembly}

In the microfluidic device, the hydrodynamic force changed with the fluidic flow rates, which, in the end, affected the assembling dynamics of the droplets. By simply changing the two phase flow rates, the 
single-layer, double-layer and multiple-layer close-packed arrangements were obtained in the confined microchannels. It was found that, in the dripping region, the microdroplets could pack into ordered single-layer lattices along the surface even at the volume fraction (the ratio of total droplets volume to the confined channel volume) of less than 0.5 . While in the jetting region, microdroplets generally assembled into close-packed double or multiple layers without experiencing the well-ordered single-layer pattern. This might be due to the stable laminar fluidic flow in the confined channel. The monodispersed droplets tended to rearrange orderly along the flow gradient.

We took the microdroplets assembly in the microfluidic device with the characteristic size of $w_{\mathrm{f}}=25 \mu \mathrm{m}, d_{\mathrm{c}}=40 \mu \mathrm{m}$ and $W_{\mathrm{a}}=400 \mu \mathrm{m}$ as an example to explore the effect of $Q_{\mathrm{o}}$ and $Q_{\mathrm{w}}$ on the microdroplets assembly. Figure 6 illustrated the microdroplets assembly configurations corresponding to the two phase volume flow rates. When the microdroplets formation was located in the transition region (as shown in Figure 3: area ii), it was difficult for the microdroplets to arrange into ordered patterns owing to the instability of the microdroplets production and fluidic flowing, as shown in Figure 6 iii(x) area. Monodispersed microdroplets were close-packed into well-ordered single-layer patterns at low flow rates for two reasons: the flow was stable to achieve ordered assembling instead of randomly arranging, and the volume fraction was not big enough for further packing according to the gentle flow. Whereas at higher flow rates, microdroplets were able to assemble into close-packed well-ordered double-layer configurations resulted from the dramatically increased volume fraction, which led to the rapid accumulation to high density packing. In addition, in the Figure 6 iii(y) area, there were no ordered arrangements observed because of the low droplet generation frequency.

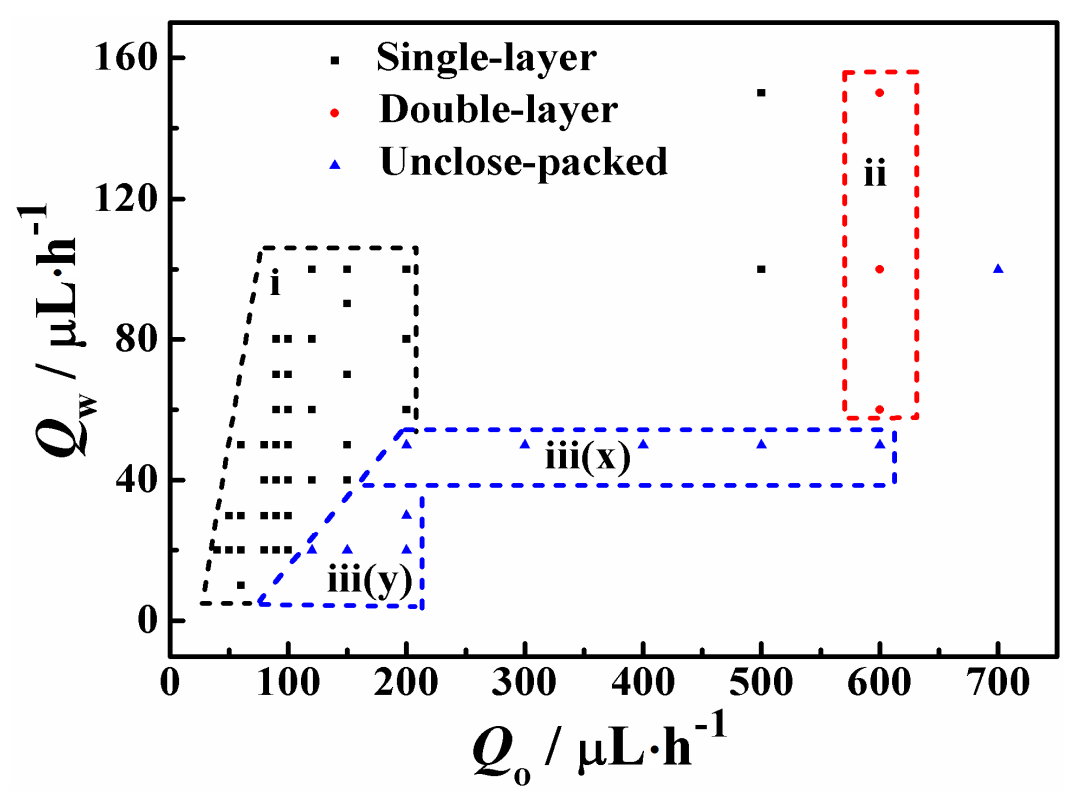

Figure 6. Diagram of the microdroplets assembly configurations as a function of two phase flow rates. The configurations were divided into: (i) close-packed well-ordered single-layer configurations, (ii) close-packed highly-ordered double-layer configurations and (iii) unclose-packed (loose or random) configurations. The microdroplets were generated in either dripping or jetting region at different flow rates. The dimensions of the flow-focusing microfluidic device were: $w_{\mathrm{f}}=25 \mu \mathrm{m}, d_{\mathrm{c}}=40 \mu \mathrm{m}$ and $W_{\mathrm{a}}=400 \mu \mathrm{m}$. Hexadecane with $2.7 \%(w / v)$ Span 80 and DI water were used as the oil phase and water phase, respectively. 
In Figure 6, there were more data points found in the bottom-left area than top-right area because of the stable droplet generation and fluidic flow at low flow rates rather than high flow rates. The fluidic flow rate not only affected the capability of creating numerous monodispersed microdroplets at a particular period of time but also determined the microdroplet assembly configurations. The precise controllability of fluidic flow in the device is critical for the microdroplets assembling into ordered configurations in the confined microchannels.

\subsubsection{Microdroplets Size Effect on the Assembly Configurations}

The crystalline lattice of droplet packing model in theory for predicting high density monodispersed droplets assembly patterns by controlling the confined channel depth or droplet size has been demonstrated by Lee et al. [10]. By changing only the depth of the microchannel $\left(d_{\mathrm{c}}\right)$ relative to a given monodispersed droplet diameter $(D)$, predictable close-packed single-, double- or multiple-layer lattices could be obtained. Hereafter, the ratio of the microchannel depth $\left(d_{\mathrm{c}}\right)$ to droplet size $(D)$ was referred to as $d_{\mathrm{c}} / D$. Miller index notation was employed to describe liquid crystallographic planes: namely (111), (110) and (100) crystal orientations. Figure 7 presented predictable close-packed well-ordered colloidal crystal configurations, expecting monodispersed droplets assembly corresponding to $d_{\mathrm{c}} / D$ values.

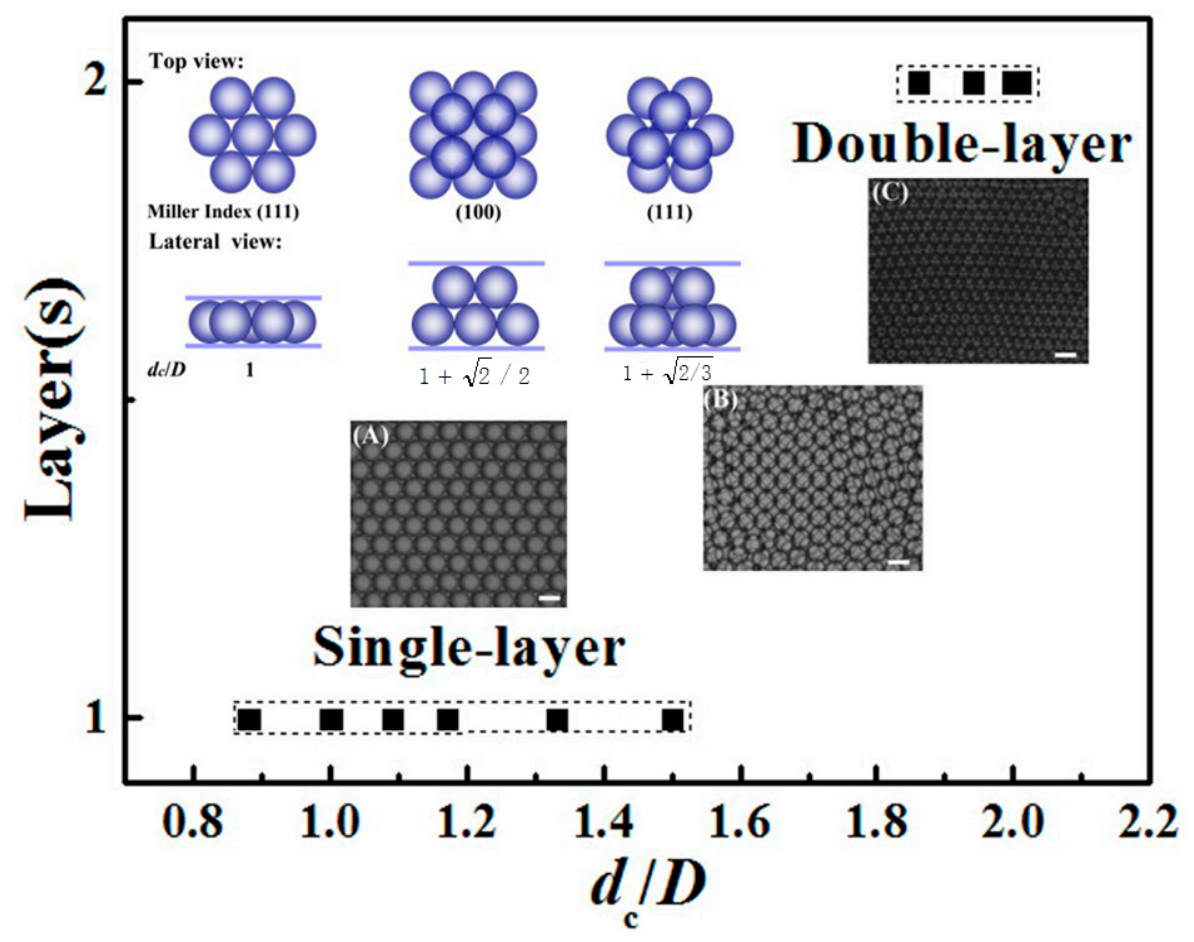

Figure 7. Diagram of droplets close-packing configurations corresponding to $d_{\mathrm{c}} / D$. The top-left insertion is the schematic drawing of droplets pattern in Miller lattice orientations. The images of (A), (B) and (C) were single-layer hexagonal (111), double-layer square (100) and double-layer hexagonal (111) configurations, respectively. Scale bars are $50 \mu \mathrm{m}$. Here, the microfluidic device was: $w_{\mathrm{f}}=25$ or $50 \mu \mathrm{m}, d_{\mathrm{c}}=40 \mu \mathrm{m}, W_{\mathrm{a}}=400 \mu \mathrm{m}$. Hexadecane with $2.7 \%(w / v)$ Span80 and DI water were used as the oil phase and water phase, respectively. 
Monodispersed microdroplets were produced in the microfluidic device with $w_{\mathrm{f}}=25$ or $50 \mu \mathrm{m}$, $d_{\mathrm{c}}=40 \mu \mathrm{m}$ and $W_{\mathrm{a}}=400 \mu \mathrm{m} . d_{\mathrm{c}} / D$ value was dynamically controlled by adjusting droplet size for a given channel depth $(40 \mu \mathrm{m})$. The well-ordered close-packed assembly configurations in common Miller index configurations were obtained at different $d_{\mathrm{c}} / D$ which were $1.11,1.67$ and 1.90 for (A), (B) and $(\mathrm{C})$ in Figure 7, respectively. Single-layer hexagonal configuration was observed for $36 \mu \mathrm{m}$ droplets assembled in the $40 \mu \mathrm{m}$ deep channel with $d_{\mathrm{c}} / D=1.11$ (A). A $d_{\mathrm{c}} / D$ value of 1.67 (24 $\mu \mathrm{m}$ droplets) favored double-layer square configuration (B). A $d_{\mathrm{c}} / D$ value of 1.90 was beneficial for $21 \mu \mathrm{m}$ droplets to assemble in double-layer hexagonal pattern (C).

Various droplet assembly configurations in the confined channel were statistically analyzed. It was found that, in the range of $d_{\mathrm{c}} / D=0.9-1.5$, the monodispersed droplets would assemble into close-packed single-layer hexagonal configurations, and the corresponding microdroplets generation was mostly in the dripping region. Whereas, when $d_{\mathrm{c}} / D$ was in the range of 1.8-2.1, monodispersed droplets would self-organize into close-packed double-layer hexagonal pattern in (111) orientation at high flow rates in the jetting region. When $d_{\mathrm{c}} / D$ was ranged from 1.5 to 1.8 , the droplet arrangements would experience transition from close-packed single-layer hexagonal (111) to double-layer square (100) configurations. Liquid droplet assembly would be better suitable for confined geometries due to their flexible mobility and ease of deformation compared to solid colloidal particles.

\subsubsection{Effect of Fluids on Droplet Generation and Assembly}

Different fluidic materials have also been investigated to explore the effect of the fluidic properties on the assembly configurations for the future selection of functional materials for different applications. Polymerizable aqueous solutions composed of NIPAM monomer (6 wt \%-25 wt \%), MBA cross-linker (1 wt \%-3 wt \%) and I 2959 photoinitiator $(0.2 \mathrm{wt} \%-3 \mathrm{wt} \%)$ were used as the water phases. The oil phases were hexadecane with Span 80 or mineral oil contained ABIL EM 90. The same microfluidic device was used. Similar phenomenon has been observed for monodispersed droplets formation and assembly, as shown in Figure S3. Differently, for the fluidic system of NIPAM aqueous solution and hexadecane with Span 80 organic solution, in a range of flow rates, much smaller microdroplets were produced and then rapidly assembled into ordered arrangement in multiple-layer pattern.

As an example, 15 wt \% NIPAM photocurable aqueous solution mixed with $3.0 \mathrm{wt} \% \mathrm{MBA}$ and 1.5 wt \% I 2959 was used as the water phase and hexadecane contained 2.7\% ( $w / v)$ Span80 was used as the oil phase. Interestingly, as shown in Figure 8, we obtained the monodispersed droplets with the diameter of only $7 \mu \mathrm{m}$ at $Q_{\mathrm{o}}=150 \mu \mathrm{L} \cdot \mathrm{h}^{-1}$ and $Q_{\mathrm{w}}=20 \mu \mathrm{L} \cdot \mathrm{h}^{-1}$. However, at the same flow rates in the same device, the droplets with diameter of $28 \mu \mathrm{m}$ were obtained using hexadecane with $2.7 \%(w / v)$ Span 80 and DI water as oil and water phase. The close-packed structure was partially in multiple-layer configuration according to the smaller droplet size. This might attribute to the effect of the interfacial tension between the two immiscible oil-water systems. The interfacial tension of the 15 wt $\%$ NIPAM aqueous solution (3.0 wt \% MBA and $1.5 \mathrm{wt} \% \mathrm{I}$ 2959)/2.7\% (w/v) Span 80 hexadecane solution was $1.62 \mathrm{mN} \cdot \mathrm{m}^{-1}$, which was ten times smaller than $15.0 \mathrm{mN} \cdot \mathrm{m}^{-1}$ for the DI water $/ 2.7 \%(w / v)$ Span 80 hexadecane solution. With lower interfacial tension, the hydrodynamic force was relatively more prominent, which could induce quicker and smaller droplets formation, and then more layers of droplets arrangement. In the future, the microdroplets composed of photocurable NIPAM aqueous solution could 
be on-line polymerized into functional microparticles, which could be potentially applied as valves, actuators or sensors.

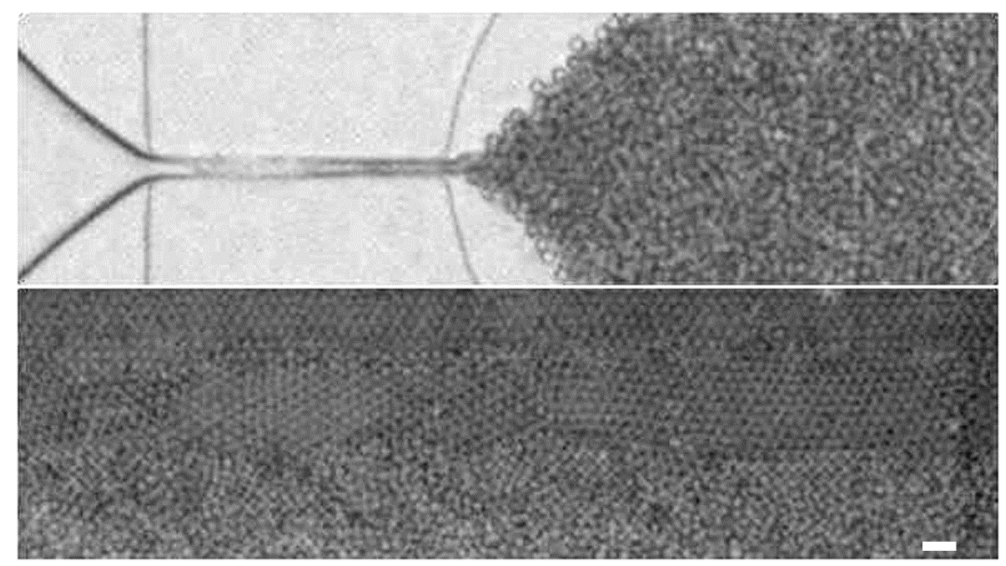

Figure 8. Optical microscopic images of the microdroplets generation and assembly multiple-layer configuration of the functional fluidic material system. The water phase was $15 \mathrm{wt} \%$ NIPAM aqueous solution mixed with $3.0 \mathrm{wt} \%$ MBA and 1.5 wt \% I 2959, and the oil phase was hexadecane contained 2.7\% (w/v) Span80. $Q_{\mathrm{o}}$ and $Q_{\mathrm{w}}$ were 150 and $20 \mu \mathrm{L} \cdot \mathrm{h}^{-1}$, respectively. The microfluidic device dimensions were $w_{\mathrm{f}}=25 \mu \mathrm{m}, d_{\mathrm{c}}=40 \mu \mathrm{m}$ and $W_{\mathrm{a}}=400 \mu \mathrm{m}$. The scale bar is $50 \mu \mathrm{m}$.

\section{Conclusions}

In conclusion, we proposed and verified a robust strategy based on microfluidics to individually create and assemble microdroplets into close-packed configurations on-demand. The droplet creation was driven by the low $\mathrm{Ca}$ number in the microfluidic device. The monodispersed droplets assembled were mainly driven by the volumetric effect, the capillarity, and the hydrodynamic force in the microchannels. Single-layer, double-layer and multiple-layer close-packed arrangements have been successfully achieved by controlling the flow rates of the two fluidic phases and the characteristic size of the microfluidic devices. The carrying capacity of the microdroplets in the microchannel was limited. The volume fraction could be tuned by the droplet size and channel geometry according to capillary force and fluidic flow rates. The exquisite control over the fluidic flow and fluidic properties was therefore the determinative factor for droplet generation and assembly in different configurations in confined microfluidic geometries. This technique could be further extended to smart nanodroplets and nanoparticles assembly in a controllable way using micro- and nanofluidics together with responsive materials, which would be highly in demand to construct functional devices for data storage, light collection and sensing applications. The precisely controlled chemical and physical properties offered by microfluidics would help us to deeply understand the self-assembly parameters.

\section{Supplementary Materials}

Supplementary materials can be accessed at: http://www.mdpi.com/2072-666X/6/9/1131/s1. 


\section{Acknowledgments}

We appreciate the financial support from the National Nature Science Foundation of China (NSFC No. 21303060). This work was also supported by Guangdong Natural Science Foundation (S2013010014418), Guangdong Talent Program (2011D039), Guangdong Innovative Research Team Program (201101D0104904202), Program for Changjiang Scholars and Innovative Research Team in University (IRT13064).

\section{Author Contributions}

Lingling Shui designed this project and Juan Wang performed the experiments. The data summary and writing of the article was mainly done by Juan Wang and Lingling Shui. Mingliang Jin and Tao He contributed to the microfluidic chip design and fabrication and results discussion. Guofu Zhou gave suggestions on the project management and conducted helpful discussion on the experimental results.

\section{Conflicts of Interest}

The authors declare no conflict of interest.

\section{References}

1. Doerk, G.S.; Cheng, J.Y.; Singh, G.; Rettner, C.T.; Pitera, J.W.; Balakrishnan, S.; Arellano, N.; Sanders, D.P. Enabling complex nanoscale pattern customization using directed self-assembly. Nat. Commun. 2014, 5. [CrossRef] [PubMed]

2. Liu, D.; Li, C.; Zhou, F.; Zhang, T.; Zhang, H.; Li, X.; Duan, G.; Cai, W.; Li, Y. Rapid synthesis of monodisperse au nanospheres through a laser irradiation-induced shape conversion, self-assembly and their electromagnetic coupling sers enhancement. Sci. Rep. 2015, 5. [CrossRef] [PubMed]

3. Singh, P.; Hossain, M.; Gurupatham, S.; Shah, K.; Amah, E.; Ju, D.; Janjua, M.; Nudurupati, S.; Fischer, I. Molecular-like hierarchical self-assembly of monolayers of mixtures of particles. Sci. Rep. 2014, 4. [CrossRef] [PubMed]

4. Stratford, K.; Henrich, O.; Lintuvuori, J.; Cates, M.; Marenduzzo, D. Self-assembly of colloid-cholesteric composites provides a possible route to switchable optical materials. Nat. Commun. 2014, 5. [CrossRef] [PubMed]

5. Miszta, K.; de Graaf, J.; Bertoni, G.; Dorfs, D.; Brescia, R.; Marras, S.; Ceseracciu, L.; Cingolani, R.; van Roij, R.; Dijkstra, M. Hierarchical self-assembly of suspended branched colloidal nanocrystals into superlattice structures. Nat. Mater. 2011, 10, 872-876. [CrossRef] [PubMed]

6. Alexeev, V.L.; Das, S.; Finegold, D.N.; Asher, S.A. Photonic crystal glucose-sensing material for noninvasive monitoring of glucose in tear fluid. Clin. Chem. 2004, 50, 2353-2360. [CrossRef] [PubMed]

7. Fudouzi, H.; Xia, Y. Photonic papers and inks: Color writing with colorless materials. Adv. Mater. 2003, 15, 892-896. [CrossRef]

8. Masuda, Y.; Itoh, T.; Koumoto, K. Self-assembly patterning of silica colloidal crystals. Langmuir 2005, 21, 4478-4481. [CrossRef] [PubMed] 
9. Asbahi, M.; Lim, K.T.; Wang, F.; Duan, H.; Thiyagarajah, N.; Ng, V.; Yang, J.K. Directed self-assembly of densely packed gold nanoparticles. Langmuir 2012, 28, 16782-16787. [CrossRef] [PubMed]

10. Hatch, A.C.; Fisher, J.S.; Pentoney, S.L.; Yang, D.L.; Lee, A.P. Tunable 3D droplet self-assembly for ultra-high-density digital micro-reactor arrays. Lab Chip 2011, 11, 2509-2517. [CrossRef] [PubMed]

11. Sackmann, E.K.; Fulton, A.L.; Beebe, D.J. The present and future role of microfluidics in biomedical research. Nature 2014, 507, 181-189. [CrossRef] [PubMed]

12. Nunes, P.S.; Mortensen, N.A.; Kutter, J.P.; Mogensen, K.B. Refractive index sensor based on a 1D photonic crystal in a microfluidic channel. Sensors 2010, 10, 2348-2358. [CrossRef] [PubMed]

13. Hayes, R.A.; Feenstra, B. Video-speed electronic paper based on electrowetting. Nature 2003, 425, 383-385. [CrossRef] [PubMed]

14. Hashimoto, M.; Mayers, B.; Garstecki, P.; Whitesides, G.M. Flowing lattices of bubbles as tunable, self-assembled diffraction gratings. Small 2006, 2, 1292-1298. [CrossRef] [PubMed]

15. Luo, R.-C.; Chen, C.-H. Structured microgels through microfluidic assembly and their biomedical applications. Soft 2012, 1, 1-23. [CrossRef]

16. Wang, J.T.; Wang, J.; Han, J.J. Fabrication of advanced particles and particle-based materials assisted by droplet-based microfluidics. Small 2011, 7, 1728-1754. [CrossRef] [PubMed]

17. Kim, J.H.; Jeon, T.Y.; Choi, T.M.; Shim, T.S.; Kim, S.-H.; Yang, S.-M. Droplet microfluidics for producing functional microparticles. Langmuir 2013, 30, 1473-1488. [CrossRef] [PubMed]

18. Shui, L.; van den Berg, A.; Eijkel, J.C. Interfacial tension controlled w/o and o/w 2-phase flows in microchannel. Lab Chip 2009, 9, 795-801. [CrossRef] [PubMed]

19. Shui, L.; van den Berg, A.; Eijkel, J.C. Scalable attoliter monodisperse droplet formation using multiphase nano-microfluidics. Microfluidics Nanofluidics 2011, 11, 87-92. [CrossRef]

20. Malekpourkoupaei, A.; Kostiuk, L.W.; Harrison, D.J. Fabrication of binary opal lattices in microfluidic devices. Chem. Mater. 2013, 25, 3808-3815. [CrossRef]

21. Shui, L.; Kooij, E.S.; Wijnperlé, D.; van den Berg, A.; Eijkel, J.C. Liquid crystallography: 3D microdroplet arrangements using microfluidics. Soft Matter 2009, 5, 2708-2712. [CrossRef]

22. Seo, M.; Nie, Z.; Xu, S.; Lewis, P.C.; Kumacheva, E. Microfluidics: From dynamic lattices to periodic arrays of polymer disks. Langmuir 2005, 21, 4773-4775. [CrossRef] [PubMed]

23. Hu, Y.; Wang, J.; Wang, H.; Wang, Q.; Zhu, J.; Yang, Y. Microfluidic fabrication and thermoreversible response of core/shell photonic crystalline microspheres based on deformable nanogels. Langmuir 2012, 28, 17186-17192. [CrossRef] [PubMed]

24. Kim, S.H.; Jeon, S.J.; Jeong, W.C.; Park, H.S.; Yang, S.M. Optofluidic synthesis of electroresponsive photonic janus balls with isotropic structural colors. Adv. Mater. 2008, 20, 4129-4134. [CrossRef]

25. Shang, L.; Shangguan, F.; Cheng, Y.; Lu, J.; Xie, Z.; Zhao, Y.; Gu, Z. Microfluidic generation of magnetoresponsive janus photonic crystal particles. Nanoscale 2013, 5, 9553-9557. [CrossRef] [PubMed] 
26. Yin, S.-N.; Wang, C.-F.; Liu, S.-S.; Chen, S. Facile fabrication of tunable colloidal photonic crystal hydrogel supraballs toward a colorimetric humidity sensor. J. Mater. Chem. C 2013, 1, 4685-4690. [CrossRef]

27. Yuet, K.P.; Hwang, D.K.; Haghgooie, R.; Doyle, P.S. Multifunctional superparamagnetic janus particles. Langmuir 2009, 26, 4281-4287. [CrossRef] [PubMed]

28. Choi, S.; Park, I.; Hao, Z.; Holman, H.-Y.N.; Pisano, A.P.; Zohdi, T.I. Ultrafast self-assembly of microscale particles by open-channel flow. Langmuir 2009, 26, 4661-4667. [CrossRef] [PubMed]

29. Park, J.I.; Nie, Z.; Kumachev, A.; Abdelrahman, A.I.; Binks, B.P.; Stone, H.A.; Kumacheva, E. A microfluidic approach to chemically driven assembly of colloidal particles at gas-liquid interfaces. Angew. Chem. 2009, 121, 5404-5408. [CrossRef]

30. Thorsen, T.; Roberts, R.W.; Arnold, F.H.; Quake, S.R. Dynamic pattern formation in a vesicle-generating microfluidic device. Phys. Rev. Lett. 2001, 86, 4163. [CrossRef] [PubMed]

31. Woodward, A.; Cosgrove, T.; Espidel, J.; Jenkins, P.; Shaw, N. Monodisperse emulsions from a microfluidic device, characterised by diffusion nmr. Soft Matter 2007, 3, 627-633. [CrossRef]

32. Xia, Y.; Whitesides, G.M. Soft lithography. Annu. Rev. Mater. Sci. 1998, 28, 153-184. [CrossRef]

33. Yobas, L.; Martens, S.; Ong, W.-L.; Ranganathan, N. High-performance flow-focusing geometry for spontaneous generation of monodispersed droplets. Lab Chip 2006, 6, 1073-1079. [CrossRef] [PubMed]

34. Utada, A.; Lorenceau, E.; Link, D.; Kaplan, P.; Stone, H.; Weitz, D. Monodisperse double emulsions generated from a microcapillary device. Science 2005, 308, 537-541. [CrossRef] [PubMed]

35. Reddy, V.; Zahn, J.D. Interfacial stabilization of organic-aqueous two-phase microflows for a miniaturized DNA extraction module. J. Coll. Interface Sci. 2005, 286, 158-165. [CrossRef] [PubMed]

36. Joanicot, M.; Ajdari, A. Droplet control for microfluidics. Science 2005, 309, 887-888. [CrossRef] [PubMed]

37. Zhao, C.-X.; Middelberg, A.P. Two-phase microfluidic flows. Chem. Eng. Sci. 2011, 66, 1394-1411. [CrossRef]

38. Nie, Z.; Seo, M.; Xu, S.; Lewis, P.C.; Mok, M.; Kumacheva, E.; Whitesides, G.M.; Garstecki, P.; Stone, H.A. Emulsification in a microfluidic flow-focusing device: Effect of the viscosities of the liquids. Microfluid. Nanofluid. 2008, 5, 585-594. [CrossRef]

39. Fu, T.; Wu, Y.; Ma, Y.; Li, H.Z. Droplet formation and breakup dynamics in microfluidic flow-focusing devices: From dripping to jetting. Chem. Eng. Sci. 2012, 84, 207-217. [CrossRef]

40. Baroud, C.N.; Gallaire, F.; Dangla, R. Dynamics of microfluidic droplets. Lab Chip 2010, 10, 2032-2045. [CrossRef] [PubMed]

(C) 2015 by the authors; licensee MDPI, Basel, Switzerland. This article is an open access article distributed under the terms and conditions of the Creative Commons Attribution license (http://creativecommons.org/licenses/by/4.0/). 